\title{
An excess of extreme TeV blazars from the Fermi LAT distribution on the voidiness parameter
}

\author{
Timur Dzhatdoev ${ }^{a,+*}$ and Emil Khalikov ${ }^{a}$ \\ ${ }^{\dagger a}$ Federal State Budget Educational Institution of Higher Education M.V. Lomonosov Moscow \\ State University, Skobeltsyn Institute of Nuclear Physics (SINP MSU), 1(2), Leninskie gory, \\ GSP-1, Moscow 119991, Russian Federation \\ ${ }^{+}$E-mail: timur1606@gmail.com
}

\begin{abstract}
Recently it was found that Fermi LAT blazars with relatively hard $(\gamma<3)$ observed spectra above $10 \mathrm{GeV}$ are predominantly located towards the directions to voids in the large scale structure and, moreover, the observed flux of $E>10 \mathrm{GeV} \gamma$-rays is usually greater for the case of such directions. This effect may be interpreted in the framework of the "electromagnetic cascade model" (T.A. Dzhatdoev et al., A\&A, 603, A59 (2017)). Namely, the secondary (cascade) component significantly enhances the observable flux in the $10-500 \mathrm{GeV}$ energy region, rendering the sources brighter in this energy range. The model predicts the existence of a new population of blazars with very hard spectra in the $10 \mathrm{GeV}-1 \mathrm{TeV}$ energy region that could be discovered in future by the CTA observatory.
\end{abstract}

35th International Cosmic Ray Conference - ICRC2017

10-20 July, 2017

Bexco, Busan, Korea

\footnotetext{
* Speaker.

$\dagger$ T.D. acknowledges the support of the Students and Researchers Exchange Program in Sciences (STEPS), the Re-Inventing Japan Project, JSPS, and the hospitality of the University of Tokyo ICRR
} 


\section{Introduction}

Robust and precise measurement of extragalactic magnetic field (EGMF) parameters would open a new window for astrophysics and cosmology [1]. Such a measurement, however, is still absent (for recent constraints on the EGMF strength $B$ and spatial structure see [2]). The typical $B$ value in voids of the large scale structure (LSS) could range from $\sim 10^{-9} \mathrm{G}[3]$ to $\sim 10^{-17}-10^{-19} \mathrm{G}$ [4]-[5], assuming a typical correlation length of $1 \mathrm{Mpc}$. An indirect method to study the EGMF by constraining the secondary (cascade) component in blazar spectra using data of orbital instruments, such as Fermi LAT [6], and Cherenkov telescopes, such as H.E.S.S. [7], MAGIC [8], and VERITAS [9], is in principle able to probe very weak large-scale magnetic fields $\left(\sim 10^{-19}-10^{-21} \mathrm{G}\right.$ [10]) and therefore attracted great attention.

In fact, the EGMF may be highly inhomogeneous, and its typical strength — strongly dependent on the particular line-of-sight. Therefore, the appearance of similar extragalactic beamed sources may be very different for such different directions. It was recently pointed out in [11] (hereafter F15) that blazars with hard observed spectra in the energy region 10-500 $\mathrm{GeV}$ are: 1) predominantly located towards the directions to voids in the LSS 2) have larger observed integral flux in this energy region. While the statistical significance of these two indications is still modest due to a limited sample used in F15 $(\sim 2.5 \sigma)$, these effects are in line with other deviations from the absorption-only model that neglects secondary emission from electron-positron pairs produced by primary very high energy (VHE, $E>100 \mathrm{GeV}$ ) $\gamma$-rays. It was shown that EBL fluctuations are not strong enough to account for such an effect (F15, [12]-[13]). In this paper we present an astrophysical interpretation of the effects considered by F15 in the framework of the intergalactic electromagnetic cascade model [14] (hereafter D17).

\section{Intergalactic electromagnetic cascade}

The most basic quantities defining the fate of $\gamma$-rays, electrons and positrons (hereafter simply "electrons") propagating through the Universe are their interaction rates $R_{\gamma}$ and $R_{e}$; for definitions see [15] (hereafter K12). The dependence of $R_{\gamma}$ and $R_{e}$ on energy $E$ for redshift $z=0$ calculated with two independent codes - ELMAG 2.02 (K12) (with two options of the EBL model - [16] (hereafter K04) and [17] (hereafter KD10)) and ECS 1.0 [18] (assuming the EBL model of [19] (hereafter G12)) - is shown in figure 1. Hereafter all calculations with the ELMAG code assume the KD10 EBL model, with the ECS code - the G12 model, unless stated otherwise. This calculation included interactions both on the extragalactic background light (EBL) and cosmic microwave background (CMB). For electron the secondary photon energy threshold parameter (defined as in K12) was set to $3 \mathrm{MeV}$. The coincidence between the results obtained with the ELMAG and ECS codes was found to be reasonable.

The dependence of $R_{\gamma}$ and $R_{e}$ on $E$ for several values of $z$, calculated with the ECS code, is shown in figure 2 separately for interactions on the EBL and CMB. Our works [20], [21] contain relevant comments on the interaction rate main features. One such feature is that at $E<100 \mathrm{TeV}$ primary $\gamma$-rays are predominantly absorbed on the EBL photons due to the fact that the EBL has much higher average photon energy $\langle\varepsilon\rangle$ than the CMB, and the $\gamma \gamma$ absorption process has an energy threshold. On the other hand, the inverse Compton (IC) rate at the energy range of interest 


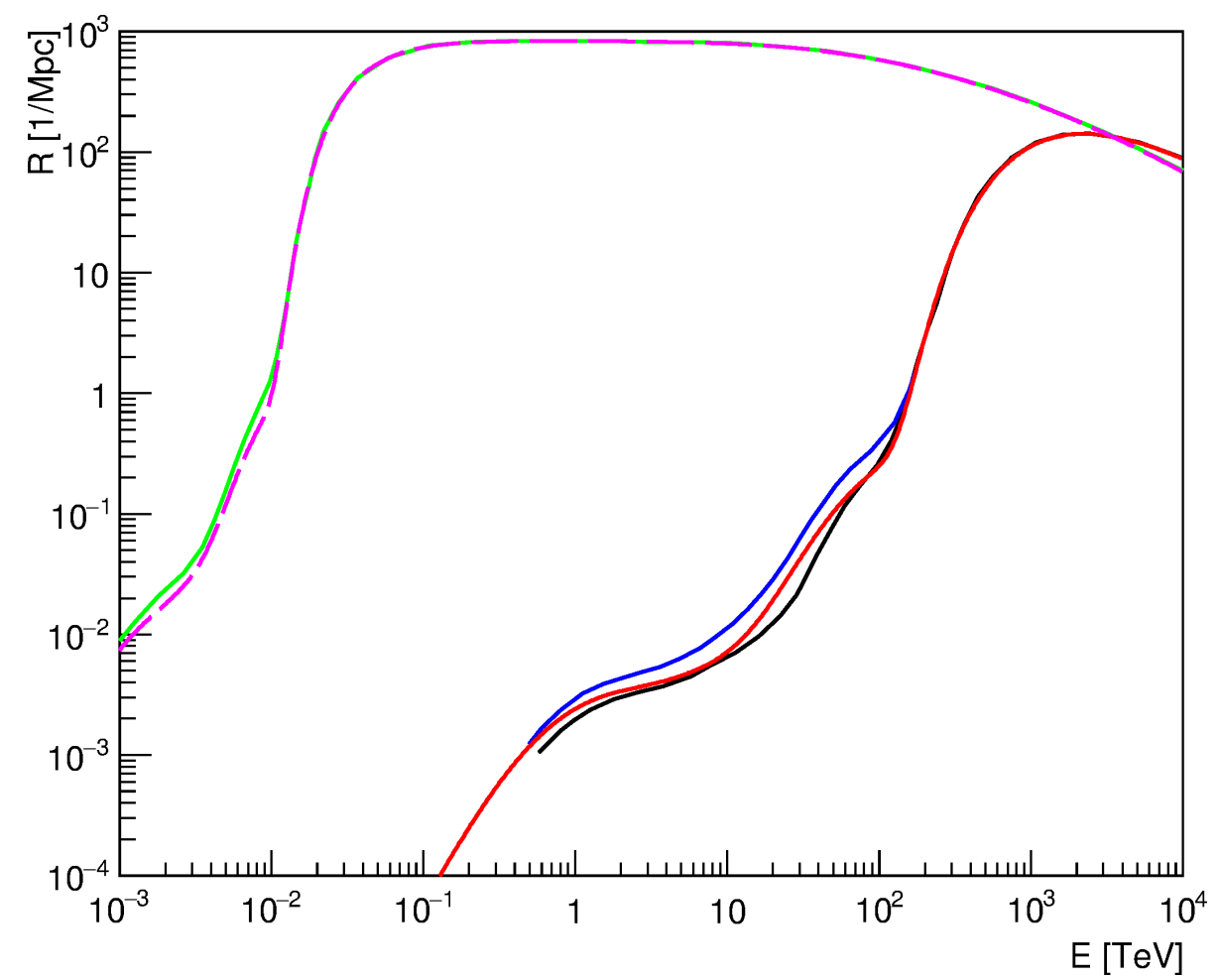

Figure 1: Comparison of interaction rates $R_{\gamma}$ and $R_{e}$ calculated with the ELMAG and ECS codes. $R_{\gamma}-$ black curve for ELMAG and KD10 EBL model, blue curve for ELMAG and K04 EBL model, red curve for ECS and G12 EBL model. $R_{e}$ - green curve for ELMAG, dashed magenta curve for ECS.

to the present paper $(E>10 \mathrm{GeV})$ is totally dominated by dense CMB photons. We also note that for rough estimates and $z<0.2$ one may safely assume $z=0$ instead, even though the dependence of $R$ on $z$ is quite strong.

In D17 we proposed a simple way to test and compare different codes intended to simulate electromagnetic (EM) cascade development in the expanding Universe. It consists in calculating observable spectra for a selection of primary monoenergetic spectra with a range of widely different energies $E_{0}$ from $1 \mathrm{TeV}$ to $1 \mathrm{PeV}$ (see figure 3). It appears that two very different regimes are apparent in this figure: the one-generation regime for $E_{0}<10 \mathrm{TeV}$ (in this case produced secondary (cascade) $\gamma$-rays practically do not experience absorption on the EBL, and the observable spectrum strongly depends on $E_{0}$ ), and the universal regime [22] for $E_{0}>100 \mathrm{TeV}$ (at least two generations of cascade $\gamma$-rays are present in this case, and the observable spectrum is practically independent on $\left.E_{0}\right)$.

For the case of primary $\gamma$-rays with an ultra-high energy (UHE, $E_{0}>1 \mathrm{EeV}$ ) the third (highenergy) regime probably sets in so that the observable spectrum becomes harder than the universal spectrum due to two effects: 1) the suppression of interaction cross sections at high values of the $M=<\varepsilon>\cdot E_{0}$ parameter 2) for high values of $M$ one of the secondaries usually carries out most of the primary particle's energy (the leading secondary effect) [22], D17. Unfortunately, for a variety of technical reasons the ELMAG and ECS codes are unable to probe robustly this high-energy regime; we leave such an investigation for future work. 


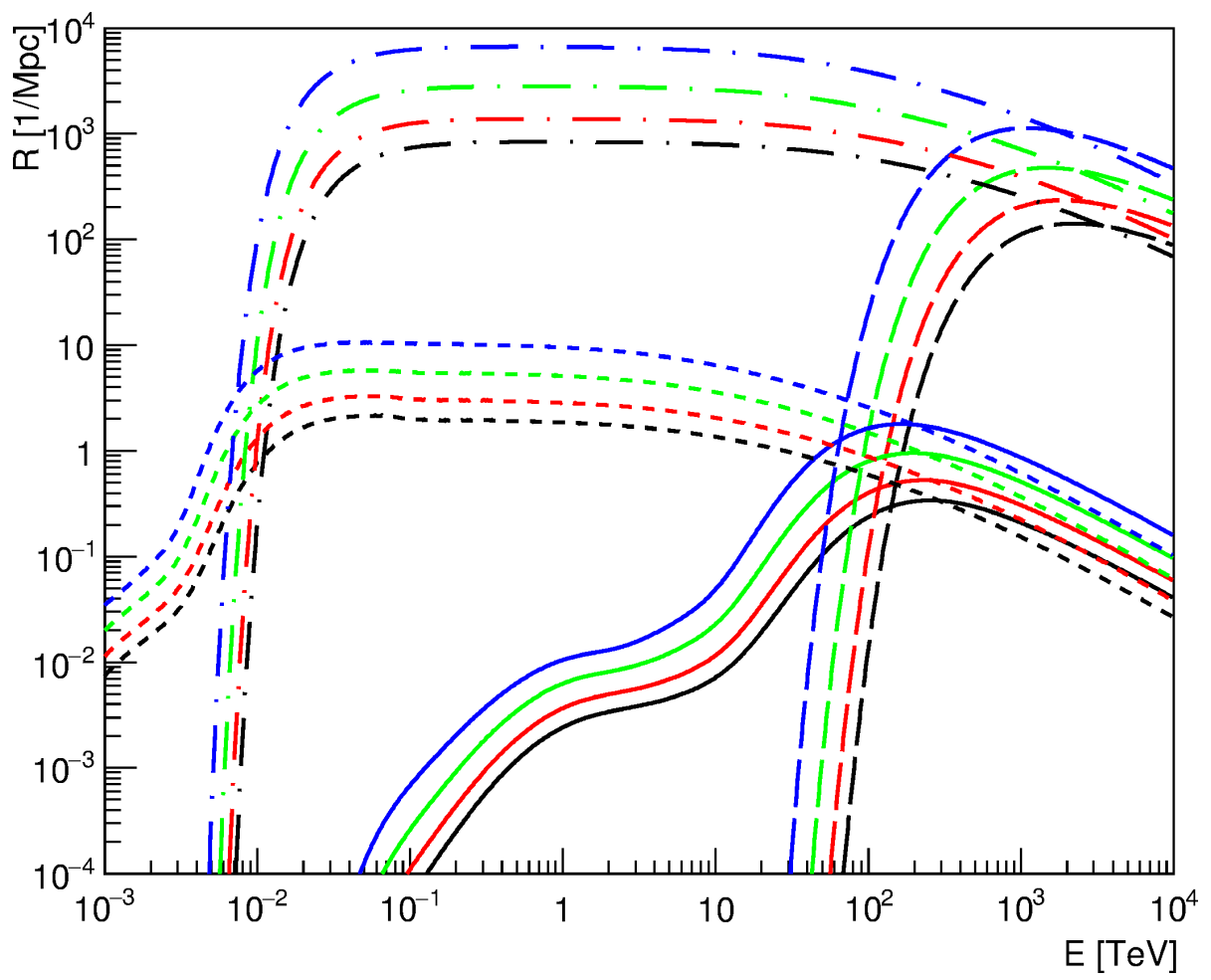

Figure 2: Interaction rates $R_{\gamma}$ (interaction on the EBL — solid curves, on the CMB - long-dashed curves) and $R_{e}$ (interaction on the EBL - short-dashed curves, on the CMB - long-dash-dotted curves). Black $z=0$, red $-z=0.186$, green $-z=0.5$, blue $-z=1$. A similar figure was published in [20].

\section{The voidiness-dependent appearance of blazars and its astrophysical interpretation}

F15 studied the dependence of several quantities such as the observed flux $F$ and the powerlaw spectral index $\gamma$ above $10 \mathrm{GeV}$, as well as the distribution of Fermi LAT hard-spectra blazars on the voidiness parameter $K$ (i.e. the fraction of line-of-sight covered by LSS voids). It was found that these blazars tend to be located towards the voids, if compared with other catalogues, such as optical sources, all Fermi LAT extragalactic sources, or a sample of uniformly distributed points. As well, $F$ appears to be typically 2-4 times greater for large values of $K>0.6$ than for $K<0.6$. Here we aim to provide the first quantitative, even if still preliminary, astrophysical interpretation of these two effects.

The first question to discuss is what extragalactic $\gamma$-ray propagation model should be chosen (for a brief review of such models, see accompanying paper [23]). As was already mentioned, the absorption-only model is unable to account for such a bright voidiness-dependent effects. An important detail of the F15 analysis is that the two mentioned effects were found at $E<300 \mathrm{GeV}$ for a sample of blazars with $z<0.36$, i.e. in the energy region with the optical depth $\tau$ well below 2. Therefore, exotic models with $\gamma$-axion-like particle (ALP) mixing [24] (see [25]-[26] for recent references) would also be unable to account for such effects, or would even predict the wrong sign of the intensity change (at low energy about $1 / 3$ of primary $\gamma$-rays typically oscillate into ALPs, so the observed intensity becomes $2 / 3$ of the one for the case of the absorption-only model). As 


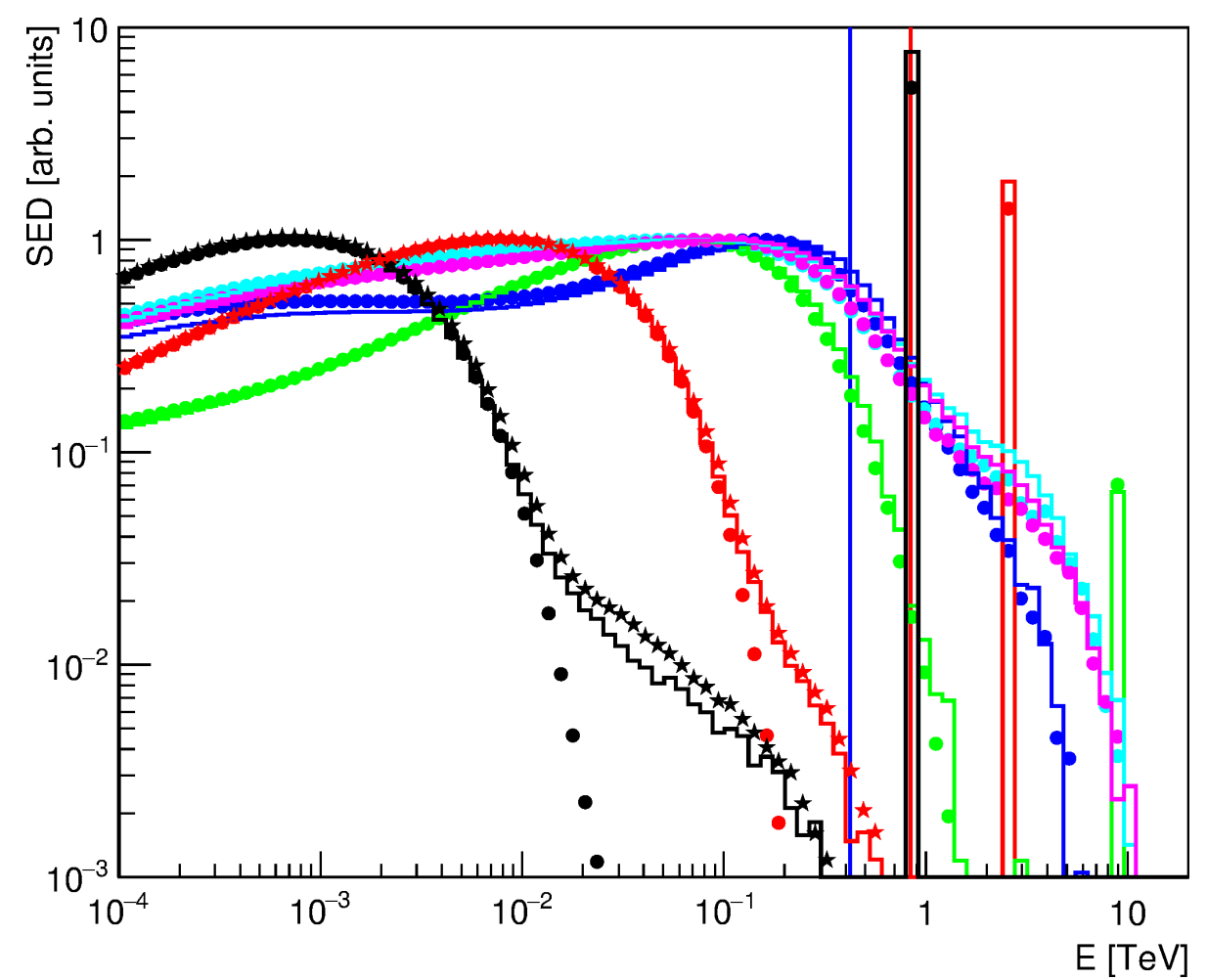

Figure 3: Observable spectra, averaged over incoming directions, for primary monoenergetic $\gamma$-rays with different $E_{0}$ [18]: black $-1 \mathrm{TeV}$, red $-3 \mathrm{TeV}$, green $-10 \mathrm{TeV}$, blue $-30 \mathrm{TeV}$, cyan $-100 \mathrm{TeV}$, magenta $-1 \mathrm{PeV}$. Histograms denote results obtained with the ELMAG code, circles - with the ECS code (IC on the CMB only), stars (for $E_{0}=1 \mathrm{TeV}$ and $3 \mathrm{TeV}$ only) - with the ECS code (IC on both CMB and EBL). Vertical red line shows the energy value $(1 \mathrm{TeV} /(1+z))$, blue line - half this value.

well, models with EM cascades initiated by primary UHE protons or nuclei were shown to have a number of difficulties [23]. The only remaining alternative is the intergalactic electromagnetic cascade model. We shall assume this model in what follows.

In figure 4 we show two fits to the SED of blazar 1ES 1218+304 - one of the brightest sources considered in F15 that also has VERITAS and Fermi LAT measurements available [27]-[28]. EM cascade simulations for these particular fits were carried out with the ELMAG code. The first fit to the observed SED was obtained assuming $B=0$ using the approach described in D17. The second fit was obtained assuming the same primary spectrum, but $B=0.1 f G$ and other EGMF parameters from [29] using the results of this last paper, as described in [23].

To understand the effects found by F15 in the framework of our model, let us first assume that the object is located towards the region of space with low voidiness, such that the cascade component in the observable spectrum is practically suppressed due to deflection and delay of cascade electrons. In this case the observable spectrum is represented by solid black curve in figure 4 . Below $500 \mathrm{GeV}$ it is very hard, and therefore practically invisible with the Fermi LAT instrument. Even contemporary Cherenkov telescopes such as H.E.S.S. would report only a marginal detection of such an object. However, the CTA array is likely to detect such a source in future.

On the other hand, assuming high value of the voidiness parameter brings the strong cascade 


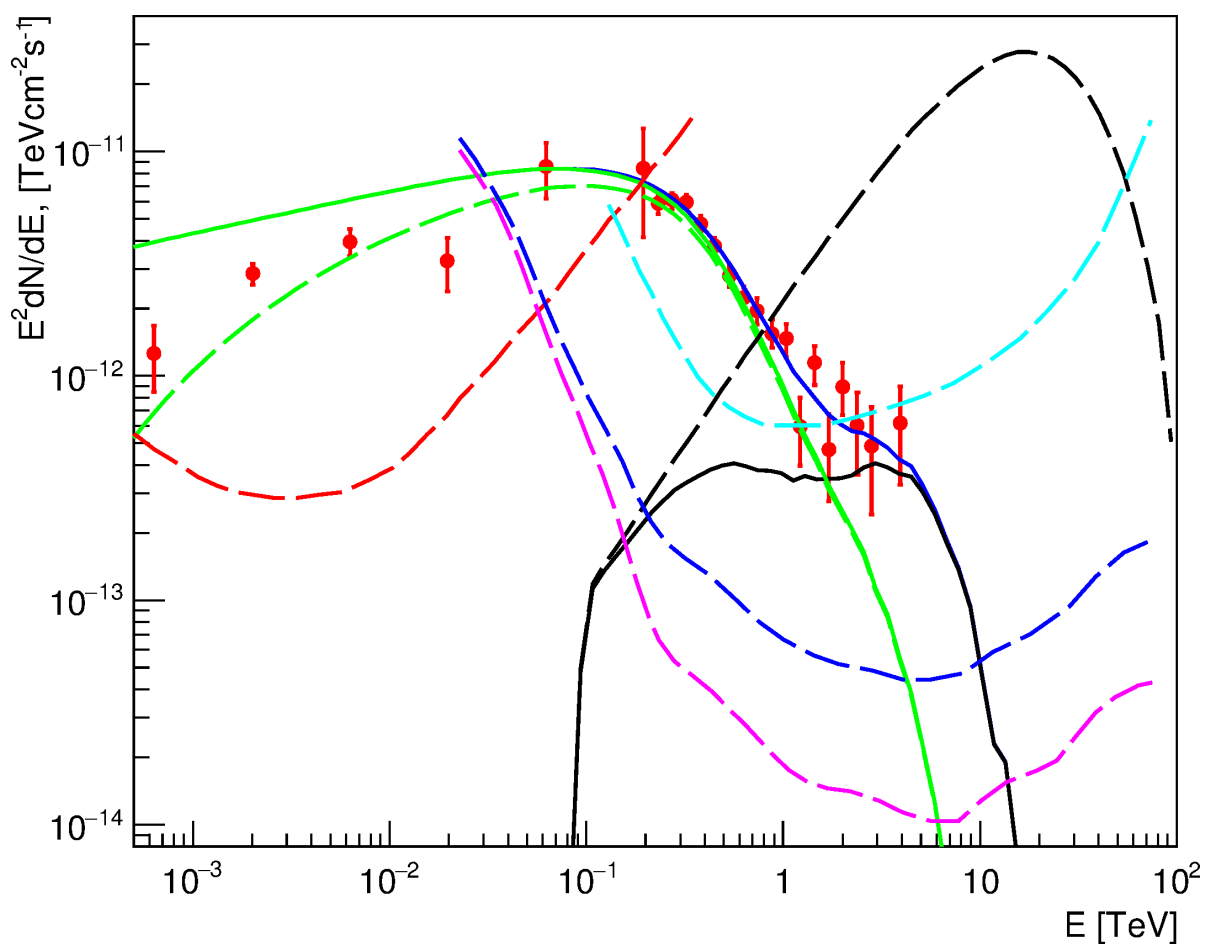

Figure 4: Two fits to the observed SED of 1ES 1218+304 [18] (red circles with statistical uncertainties). Solid blue curve denotes the fit to the VERITAS SED asuming $B=0$; the corresponding intrinsic $\gamma$-ray spectrum is denoted by dashed black curve, its attenuated component — by solid black curve, cascade component - by solid green curve. Dashed green curve denotes the second option for the cascade component assuming $B=10^{-16} \mathrm{G}$. Differential sensitivity curves for various experiments are also shown [30]: dashed red line Fermi-LAT sensitivity (10 years), dashed cyan line - H.E.S.S. sensitivity (100 hours), dashed blue line CTA sensitivity (100 hours), dashed magenta line — CTA sensitivity (1000 hours).

component into the play: in this case Fermi LAT, as well as H.E.S.S. and CTA, would be able to see bright flux of cascade $\gamma$-rays that typically have a spectrum with a power-law index $(d N / d E)$ from 1 to 2 in the 1-100 GeV energy region, depending on energy, EGMF parameters and observation conditions. This finding may explain the appearance of strong flux of $\gamma$-rays from the void-reach regions of the extragalactic space, typically with power-law index 1.5-2.2, observed by F15. The other effect of F15, i.e. the distribution of the number of detected sources on the voidiness parameter, is also nicely explained by the same model, considering a limited sensitivity of the Fermi LAT experiment.

Finally, now we are in position to make an interesting, even if dangerous, prediction that could be used in future to test our model. Namely, we predict that CTA will unveil a new population of exteme $\mathrm{TeV}$ blazars with very hard intrinsic spectra in the GeV-TeV energy range, most of them located towards the void-free regions of the extragalactic space. It is especially reassuring that several such relatively bright sources may have been already observed by [31]. On the other hand, similar objects located towards large-scale voids may display softer spectra and have much higher intensity in the $10 \mathrm{GeV}-1 \mathrm{TeV}$ energy region due to the appearance of the cascade component. In this latter case, a magnetically broadened pattern around such sources might be observed, as was indicated by [32], depending on the EGMF parameters (see also [23] for a few additional 
comments).

\section{Conclusions}

We have briefly considered the typical features of EM cascades in extragalactic photon fields, and were able to find a natural interpretation for the two voidiness-dependent effects of [11] in the framework of the intergalactic electromagnetic cascade model. The model presented here has a definite prediction: there should exist a new population of hard-spectra blazars that in future could be discovered by the CTA observatory. Additionally, a possible identification of a magneticallybroadened pattern around some sources together with variability studies could contribute to the testing of the proposed model.

\section{References}

[1] A. Neronov \& Ie. Vovk, Evidence for Strong Extragalactic Magnetic Fields from Fermi Observations of TeV Blazars, Science, 328 (2010) 73 [astro-ph/1006.3504]

[2] T.A. Dzhatdoev el al., Testing models of extragalactic $\gamma$-ray propagation using observations of extreme blazars in GeV and TeV energy ranges, J. Phys.: Conf. Ser., 798 (2017) 012002 [astro-ph/1701.06827]

[3] M.S. Pshirkov et al., New Limits on Extragalactic Magnetic Fields from Rotation Measures, Phys. Rev. Lett., 116 (2016) 191302 [astro-ph/1504.06546]

[4] C.D. Dermer et al., Time delay of cascade radiation for TeV blazars and the measurement of the intergalactic magnetic field, ApJ Lett., 733 (2011) L21 [astro-ph/1011. 6660]

[5] A.M. Taylor et al., Extragalactic magnetic fields constraints from simultaneous GeV-TeV observations of blazars, $A \& A, \mathbf{5 2 9}$ (2011) A144 [astro-ph/1101.0932]

[6] W. B. Atwood et al, The Large Area Telescope on the Fermi Gamma-ray Space Telescope Mission, ApJ, 697 (2009) 1071 [astro-ph/ 0902 .1089]

[7] J.A Hinton, The status of the HESS project, New Astron. Rev., 48 (2004) 331 [astro-ph / 0403052 ]

[8] E. Lorenz, Status of the 17 m diameter MAGIC telescope, New Astron. Rev., 48 (2004) 339

[9] F. Krennrich et al., VERITAS: the Very Energetic Radiation Imaging Telescope Array System, New Astron. Rev., 48 (2004) 345 [astro-ph / 0108478 ]

[10] A. Neronov \& D.V. Semikoz, Sensitivity of $\gamma$-ray telescopes for detection of magnetic fields in the intergalactic medium, Phys. Rev. D, 80 (2009) 123012 [astro-ph/0910.1920]

[11] A. Furniss et. al., A correlation between hard gamma-ray sources and cosmic voids along the line of sight, MNRAS, 446 (2015) 2267 [astro-ph/1407. 6370]

[12] H. Abdalla \& M. Boettcher, EBL Inhomogeneity and Hard-Spectrum Gamma-Ray Sources, ApJ, 835, (2017) 237 [astro-ph/1701.00956]

[13] A.M. Kudoda \& A. Faltenbacher, Effects of spatial fluctuations in the extragalactic background light on hard gamma-ray spectra, MNRAS, 467 (2017) 2896 [astro-ph/1702.03211]

[14] T.A. Dzhatdoev et al., Electromagnetic cascade masquerade: a way to mimic $\gamma$-axion-like particle mixing effects in blazar spectra, A\&A, $\mathbf{6 0 3}$ (2017) A59 [astro-ph/1609. 01013] 
[15] M. Kachelriess et al., ELMAG: A Monte Carlo simulation of electromagnetic cascades on the extragalactic background light and in magnetic fields, Comp. Phys. Comm., 183 (2012) 1036 [astro-ph/1106.5508]

[16] T.M. Kneiske et al., Implications of cosmological gamma-ray absorption. II. Modification of gamma-ray spectra, A\&A, 413 (2004) 807 [astro-ph/0309141]

[17] T.M. Kneiske \& H. Dole, A lower-limit flux for the extragalactic background light, A\&A, 515 (2010) A19 [astro-ph/1001.2132]

[18] T.A. Dzhatdoev, The intergalactic electromagnetic cascade solution for the anomalies from $\gamma$-ray blazar observations (2017) [ast ro-ph/1705.05360]

[19] R.C. Gilmore et al., Semi-analytic modelling of the extragalactic background light and consequences for extragalactic gamma-ray spectra, MNRAS, 422 (2012) 3189 [astro-ph/1104 . 0671 ]

[20] T.A. Dzhatdoev et al., The VHE anomaly in blazar spectra and related phenomena, EPJ Web of Conferences, 145 (2017) 17002 [astro-ph/1701.07293]

[21] T.A. Dzhatdoev, On conservative models of "the pair-production anomaly" in blazar spectra at Very High Energies, J. Phys.: Conf. Ser., 632 (2015) 012035 [astro-ph/1503. 04860 ]

[22] V. Berezinsky \& O. Kalashev, High-energy electromagnetic cascades in extragalactic space: Physics and features A110, Phys.Rev.D, 94 (2016) 023007 [astro-ph/1603.03989]

[23] T.A. Dzhatdoev et al., Extragalactic $\gamma$-ray propagation: beyond the absorption-only model, these proceedings (2017)

[24] G. Raffelt \& L. Stodolsky, Mixing of the photon with low-mass particles, Phys. Rev. D, 37 (1988) 1237

[25] A. Kartavtsev et al., Extragalactic photon-ALP conversion at CTA energies, JCAP, 01 (2017) 024 [astro-ph/1611.04526]

[26] D. Montanino et al., Enhancing the spectral hardening of cosmic TeV photons by the mixing with axion-like particles in the magnetised cosmic web (2017) [astro-ph/1703 . 07314]

[27] A.S. Madhavan for the VERITAS Collaboration, VERITAS Long-Term Observations of Hard Spectrum Blazars (2013) [astro-ph/1307 . 7051]

[28] F. Oikonomou et al., Synchrotron pair halo and echo emission from blazars in the cosmic web: application to extreme TeV blazars, A\&A, 568 (2014) A110 [astro-ph/1406. 6075]

[29] R. Alves Batista, Electromagnetic Cascades as Probes of Cosmic Magnetism (2017) [astro-ph/1704.05137]

[30] S. Funk \& J.A. Hinton, Comparison of Fermi-LAT and CTA in the region between 10-100 GeV, APh, 43 (2013) 348 [astro-ph/1205.0832]

[31] B. Arsioli \& Y.-L. Chang, Searching for $\hat{I}_{3}$-ray signature in WHSP blazars - Fermi-LAT detection of 150 excess signal in the 0.3-500 GeV band, A\&A, 598 (2017) A134 [astro-ph/1609.08501]

[32] W. Chen et al., Search for GeV $\gamma$-Ray Pair Halos Around Low Redshift Blazars, Phys.Rev.Lett., 115 (2015) 211103 [astro-ph/1410.7717] 